\title{
Computation of Nash equilibria in finite games: introduction to the symposium
}

\author{
Bernhard von Stengel
}

Published online: 24 March 2009

(C) Springer-Verlag 2009

This is a collection of ten invited articles on the computation of Nash equilibria in finite games. Three (by Ruchira Datta, Jean-Jacques Herings and Ronald Peeters, and Tim Roughgarden) are longer surveys, the others present new research. All contributions attempt to make the material accessible and interesting to the non-specialist. The collection reflects in equal measure the state of research, as well as providing an exposition of the main ideas and questions of the field. The articles are also bridged by a unity of ideas that appear in many places.

The topic of this collection is a computational problem: given a game in some finite description, find its Nash equilibria; or, a more modest task: find one Nash equilibrium of the game. These questions pose highly interesting challenges for economics, mathematics, and computer science.

For the applied economist, noncooperative games are a modeling tool, with the Nash equilibrium as the central solution concept. Interpreting and discussing a Nash equilibrium is essential for the model, but the equilibrium has to be found first, which often takes a substantial amount of the analysis. A computer program for this analysis should save time and allow for more detailed modeling.

Why should the applied economist, as a modeler and user of game theory, care about algorithms? It seems easy just to convert the equilibrium problem to a suitable optimization problem, run some mathematical software on it, and use the solution. The problem is that such a "black box algorithm" is unlikely to work on all but the smallest cases. Any algorithm that "scales" reasonably well in allowing for more detailed games as input, and still finishes in reasonable time, must exploit the mathematical structure of the problem.

B. von Stengel $(\varangle)$

Department of Mathematics, London School of Economics, London WC2A 2AE, UK

e-mail: stengel@nash.lse.ac.uk 
The mathematical structure of Nash equilibria is described and used in many of the papers in this collection: polyhedral geometry for two-player games, polynomial algebra for multi-player games, and the topology of the equilibrium correspondence between games and mixed-strategy profiles that underlies homotopy and other algorithms for finding equilibria.

Like many other topics in mathematical economics, equilibrium computation is a source of beautiful mathematical problems. These problems deserve attention from the perspective of good mathematical taste, and are most satisfactorily understood and solved in that way.

For the computer scientist, equilibrium computation belongs to the booming field of algorithmic game theory which, half a century after John von Neumann pioneered both game theory and software-driven computing, brings these two areas back together. To a large extent, algorithmic game theory is motivated by the advent of the internet as the new computing environment. Game theory is the method of choice to study its economic implications and decentralized growth and use.

As Christos Papadimitriou writes in his enthusiastic foreword to a recent textbook on algorithmic game theory (Nisan, Roughgarden, Tardos, and Vazirani 2007), equilibrium computation has been one of the earliest research goals of algorithmic game theory. One of the most basic questions is: given a bimatrix game with integer payoffs, find one Nash equilibrium of the game. In a spectacular sequence of papers (summarized in Daskalakis, Goldberg, and Papadimitriou 2006, and Chen and Deng 2006), it was recently shown that this problem is "PPAD-complete", that is, in a certain sense "as hard" as solving many other, seemingly harder, problems related to equilibria, for example finding an approximate fixed point of a Brouwer function. In his contribution, Roughgarden (2010) explains this result and many others.

We give a brief outline of the articles in alphabetical order of the authors.

In Avis et al. (2010), David Avis, Gabe Rosenberg, Rahul Savani, and Bernhard von Stengel treat the problem of finding all equilibria of a two-player game in strategic form. The presented methods have the potential to be extended to more general game descriptions, for example games in extensive form. The paper explains two known algorithms and some extensions, one of them based on vertex enumeration with "lexicographic reverse search" (Avis 2006), the other the "enumeration of extreme equilibria” by Audet, Hansen, Jaumard, and Savard (2001).

The basic "best response condition" for mixed-strategy equilibria due to Nash (1951) states that a mixed strategy is a best response to the profile of the strategies of the other players if and only if each pure strategy that is played with positive probability is a best response. The payoffs to the best-response pure strategies are maximal and therefore equal to each other, which leads to equations and inequalities; these are linear in a two-player game. The stage before solving these constraints is combinatorial: what is the set of pure strategies that a player should mix? Avis et al. explain their algorithms using the known "best response polyhedra" and their face structure, and report on computational experiments.

An algorithm that only finds at least one equilibrium, not all of them, is much more likely to finish in reasonable time for larger games than an algorithm that finds all equilibria. The classic algorithm to find one equilibrium of a bimatrix game is due to Lemke and Howson (1964). It is one of the methods compared by Anne Balthasar in 
Balthasar (2010). The Lemke-Howson algorithm has been the inspiration for many other methods that follow a path of strategy profiles that are "almost" equilibria. The algorithm is started by considering a pure strategy of one player, for definiteness say row 1 of the row player, and the (generically unique) pure best response of the other player. Unless this is already an equilibrium, it is not row 1 that is, in turn, a best response, but some other row whose probability is now increased, until an additional column becomes a best response, and is again increased; in this process, pure strategies may also be omitted as best responses when they reach zero probability. This continues until row 1 is no longer played or becomes a best response, resulting in an equilibrium.

A more flexible algorithm due to van den Elzen and Talman (1991) has an arbitrary mixed strategy pair as a starting point which is used throughout the computation for reference. Balthasar observes that it is not identical to the Lemke-Howson method when started from the same pure strategy pair. However, both algorithms are special cases of the much more general Global Newton method due to Govindan and Wilson (2003). Moreover, the latter generalizes the "tracing procedure" for games with any number of players. In addition, despite its flexibility, there are games where certain equilibria will not be found generically by the van den Elzen-Talman algorithm.

In her survey article (Datta 2010), Ruchira Datta explains computational methods based on computer algebra that find Nash equilibria of a game with any finite number of players. Nash's best-response condition imposes equality constraints on the expected payoffs to a player for the pure strategies in his equilibrium support. If the game has more than two players, multiple equations of this kind give rise to equations with polynomials of arbitrarily high degree. Combined with the inequalities for the unplayed strategies, a game defines a semi-algebraic set, defined by the solutions to equations and inequalities between polynomials with rational coefficients. In an earlier important paper, Datta (2003) has shown that any semi-algebraic set can be encoded as the set of Nash equilibria of a suitable game. This illustrates the difficulty of the problem of finding Nash equilibria, but also demonstrates that computer systems for manipulating polynomial equations are the adequate tool for it.

Datta describes how to construct certain special games and explains how to find all the complex roots of the corresponding polynomial systems, including all the Nash equilibria. These special solutions can then be used with polyhedral homotopy continuation to find all the complex roots of the polynomial systems for arbitrary generic games. "Gröbner bases" are useful for solving these polynomial systems and for obtaining geometric information about how the solution set varies with the payoff functions. Datta takes the reader carefully through each step in this development, and illustrates the use of existing computer algebra systems with specific examples.

In Govindan and Wilson (2010), Hari Govindan and Bob Wilson describe a decomposition algorithm for finding an approximate equilibrium of an $N$-player game. A central coordinator is introduced as an additional player to an $N$-player game, who receives a mixed strategy from each player and suggests a new profile of strategies back to them. An equilibrium is reached when the player's responses agree with the strategies proposed. The computational advantage of this decomposition is that it replaces the multilateral interaction among the $N$ players by $N$ bilateral interactions, one between each player and the coordinator. Two-player interactions are 
algorithmically much more amenable, because any path-following is along straight lines, in discrete "pivoting" steps that jump from one end of a line segment to the other.

Govindan and Wilson use an elegant method inspired by the triangulation of a cube in arbitrary dimension due to Kuhn (1960). In turn, the triangulation itself is used to triangulate a simplex, or any product of simplices such as the product of the players' strategy sets. Based on this triangulation, an equilibrium strategy profile of the decomposed game is found for the desired accuracy. The path-following along simplices in the triangulation is a variant of the algorithm by Lemke and Howson (1964) and its extension to more general linear complementarity problems (Lemke 1965; Eaves 1971).

Jean-Jacques Herings and Ronald Peeters give a survey of homotopy methods to compute equilibria (Herings and Peeters 2010). The idea behind the homotopy principle is that the given game that defines the equilibrium problem is transformed to another "starting" game where an equilibrium is easily found. This starting game is then continuously transformed into the original game while maintaining the equilibrium. This equilibrium follows a path that ends at an equilibrium of the given game. The fixed-point theorem of Browder (1960) asserts that this principle works. Herings and Peeters explain carefully the mathematical assumptions and the unifying view provided by the homotopy principle. They use this view to present algorithms for bimatrix games and for games with any number of players, including their own important method (Herings and Peeters 2001).

Ravi Kannan and Thorsten Theobald provide a very interesting hierarchy of bimatrix games (Kannan and Theobald 2010). It begins at zero-sum games, which are easy to solve, and ends at general bimatrix games. In each hierarchy class, the payoff matrix of one player can be generic, but the payoff matrix of the other player is related to the first via the fixed rank of the sum of the two payoff matrices. This rank is zero for zero-sum games, and maximal for general bimatrix games. Kannan and Theobald show that for games with constant rank, an $\varepsilon$-approximate equilibrium can be found in time that is polynomial in the size of the game and in $1 / \varepsilon$. Their paper raises also many intriguing open problems related to this hierarchy of games.

Peter Bro Miltersen and Troels Bjerre Sørensen show how to compute a quasiperfect equilibrium of a two-player game in extensive form in Miltersen and Sørensen (2010). Quasi-perfect equilibria are an attractive refinement concept because they are sequential (Kreps and Wilson 1982) and normal-form perfect. For extensive games, methods that use the strategic form are not suitable for larger games because a player typically has exponentially many strategies in the size of the game. A method to compute directly with the extensive game was suggested by Wilson (1972), and employed by Koller and Megiddo (1992) in a polynomial-time algorithm that finds an equilibrium of a zero-sum extensive game. A better strategic description that has the same size as the game tree had been found by Romanovskii (1962). However, this work was overlooked in the English-speaking literature and rediscovered by von Stengel (1996) as the "sequence form" of an extensive game. Miltersen and Sørensen adapt the algorithm of Lemke (1965) to the sequence form with a symbolic perturbation that leads to optimal behavior even off the equilibrium path. This is important, for example, for applications in artificial intelligence where a poker playing program should exploit mistakes of an opponent who does not play optimally. 
Tim Roughgarden's survey (Roughgarden 2010) is directed at readers with a background in economics. Computational complexity deals with the intrinsic difficulty of solving a computational problem. A main tool is that of a polynomial-time reduction which allows to show that one problem is at least as hard as another one. This is vividly and clearly explained in the article by means of examples from economics. Roughgarden gives a nonstandard but accessible definition of the important complexity class NP (which gives rise to the term "NP-complete"). Namely, problems in NP are defined as search problems rather than mere decision problems: the computation is meant to provide a solution to the problem (or state that none exists) rather than merely say "yes" or "no" whether a solution exists. This is important for finding equilibria which always exist, so that the decision problem is uninteresting.

In his very original and interesting survey, Roughgarden explains the role of equilibrium computation in two complexity classes of search problems: finding a purestrategy equilibrium in a congestion game belongs to the class PLS for "polynomial local search", and finding a mixed equilibrium of a bimatrix game to the class PPAD for "polynomial parity argument with direction" (an example of such a parity argument is the Lemke-Howson algorithm, which was extended to $N$-player games by Wilson 1971). Moreover, both of these equilibrium problems are complete for the respective class. The PPAD-completeness of finding an approximate Nash equilibrium of a threeplayer game (Daskalakis et al. 2006) and even of a two-player game (Chen and Deng 2006) was an unexpected recent result that received much attention. Roughgarden's paper is a good entry point to that literature.

Eilon Solan and Nicolas Vieille (Solan and Vieille 2010) are concerned with the computability of strategies in stochastic games. They extend work by Chatterjee, Majumdar, and Henzinger (2008) who have shown that the value of zero-sum games can be found in exponential time. The paper is a technical deconstruction of the proof by Mertens and Neyman (1981) that shows that stochastic games have a value. It shows that the $\varepsilon$-optimal strategy constructed in the proof of Mertens and Neyman is computable. Solan and Vieille construct formulas that approximate the "Puiseux series" representation of uniform strategies in discounted games by constructing the leading exponent and leading coefficient of the Pusieux series. Their main algorithmic tool is the theory of the reals, which is decidable. This article should stimulate further interest in computational issues in the theory of stochastic games.

In Turocy (2010), Ted Turocy applies the concept of logit quantal response equilibrium (McKelvey and Palfrey 1995) to games in extensive form. A logit quantal response equilibrium is parameterized by a scalar precision parameter. Varying this parameter defines a branch of the quantal response equilibrium correspondence. The limit of any such branch, as the parameter tends to infinity, is a Nash equilibrium of the game. Turocy shows that this can be used to compute a sequential equilibrium (Kreps and Wilson 1982) of an extensive game. The computational performance is documented by a number of examples. Quantal response equilibria have been used to explain the behavior of subjects in experiments. The Gambit game-solving software (McKelvey, McLennan, and Turocy 2008) was initiated by the late Richard McKelvey and by Andy McLennan in order to find equilibria of games used in such experiments. Ted Turocy is currently the main programmer and maintainer of Gambit. His article is a most fitting theoretical contribution to the subject. 
A number of thanks and apologies are in order at the conclusion of this editorial project. First, my apologies to those working in the field who have not been invited to contribute a paper. Given the space restrictions, this was necessary to achieve a certain breadth and variety of the contributions. I hope these colleagues will enjoy reading the articles in this collection.

I thank Roko Aliprantis for inviting and trusting me to manage this project. The authors have to be thanked for their outstanding cooperation and their patience. Early contributors had to wait for more than a year before getting feedback on their revised articles, and then responded gracefully and in time for the final deadline. I thank the many unnamed referees for their detailed and helpful responses and comments, some of them at very short notice. I would also like to take this opportunity to thank Bob Wilson, who is not only a pioneer of auction theory and game theory but also of equilibrium computation. Bob Wilson is a phenomenal supporter of young researchers in their careers, including mine, and of many much younger authors of the articles represented here.

\section{References}

Audet, C., Hansen, P., Jaumard, B., Savard, G.: Enumeration of all extreme equilibria of bimatrix games. SIAM J Sci Comput 23, 323-338 (2001)

Avis, D.: User's Guide for lrs (2006). http://cgm.cs.mcgill.ca/ avis

Avis, D., Rosenberg, G., Savani, R., von Stengel, B.: Enumeration of Nash equilibria for two-player games. Econ Theory 42 (2010, this issue). doi:10.1007/s00199-009-0449-X

Balthasar, A.: Equilibrium tracing in strategic-form games. Econ Theory 42 (2010, this issue). doi:10.1007/ s00199-009-0442-4

Browder, F.E.: On continuity of fixed points under deformations of continuous mappings. Summa Bras Math 4, 183-191 (1960)

Chatterjee, K., Majumdar, R., Henzinger, T.A.: Stochastic limit-average games are in EXPTIME. Int J Game Theory 37, 219-234 (2008)

Chen, X., Deng, X.: Settling the complexity of two-player Nash equilibrium. In: Proc. 47th Ann. Symp. Foundations of Computer Science (FOCS), pp. 261-270 (2006)

Daskalakis, C., Goldberg, P.W., Papadimitriou, C.H.: The complexity of computing a Nash equilibrium. In: Proc. 38th Ann. ACM Symp. Theory of Computing (STOC), pp. 71-78 (2006)

Datta, R.S.: Universality of Nash equilibria. Math Oper Res 28, 424-432 (2003)

Datta, R.S.: Finding all Nash equilibria of a finite game using polynomial algebra. Econ Theory $\mathbf{4 2}$ (2010, this issue). doi:10.1007/s00199-009-0447-z

Eaves, B.: The linear complementarity problem. Manage Sci 17, 612-634 (1971)

Govindan, S., Wilson, R.: A global Newton method to compute Nash equilibria. J Econ Theory 10, 65-86 (2003)

Govindan, S., Wilson, R.: A decomposition algorithm for $N$-player games. Econ Theory 42 (2010, this issue). doi:10.1007/s00199-009-0434-4

Herings, P.J.J., Peeters, R.: A differentiable homotopy to compute Nash equilibria of $n$-person games. Econ Theory 18, 159-185 (2001)

Herings, P.J.J., Peeters, R.: Homotopy methods to compute equilibria in game theory. Econ Theory 42 (2010, this issue). doi:10.1007/s00199-009-0441-5

Kannan, R., Theobald, T.: Games of fixed rank: a hierarchy of bimatrix games. Econ Theory 42 (2010, this issue). doi:10.1007/s00199-009-0436-2

Koller, D., Megiddo, N.: The complexity of two-person zero-sum games in extensive form. Games Econ Behav 4, 528-552 (1992)

Kreps, D.M., Wilson, R.: Sequential equilibria. Econometrica 50, 863-894 (1982)

Kuhn, H.: Some combinatorial lemmas in topology. IBM J Res Dev 4, 518-524 (1960)

Lemke, C.E.: Bimatrix equilibrium points and mathematical programming. Manage Sci 11, 681-689 (1965) 
Lemke, C.E., Howson, J.T. Jr.: Equilibrium points of bimatrix games. J Soc Indust Appl Math 12, 413-423 (1964)

McKelvey, R.D., Palfrey, T.R.: Quantal response equilibria for normal form games. Games Econ Behav 10, 6-38 (1995)

McKelvey, R.D., McLennan, A.M., Turocy, T.L.: Gambit: Software Tools for Game Theory (2008). http:// gambit.sourceforge.net

Mertens, J.F., Neyman, A.: Stochastic games. Int J Game Theory 10, 53-66 (1981)

Miltersen, P.B., Sørensen, T.B.: Computing a quasi-perfect equilibrium of a two-player game. Econ Theory 42 (2010, this issue). doi:10.1007/s00199-009-0440-6

Nash, J.F.: Non-cooperative games. Ann Math 54, 286-295 (1951)

Nisan, N., Roughgarden, R., Tardos, É., Vazirani, V. (eds.): Algorithmic Game Theory. Cambridge: Cambridge University Press (2007)

Romanovskii, I.V.: Reduction of a game with complete memory to a matrix game. Doklady Akademii Nauk SSSR 144:62-64 (1962) (English translation: Sov Math 3:678-681)

Roughgarden, T.: Computing equilibria: a computational complexity perspective. Econ Theory $\mathbf{4 2}$ (2010, this issue). doi:10.1007/s00199-009-0448-y

Solan, E., Vieille, N.: Computing uniformly optimal strategies in two-player stochastic games. Econ Theory 42 (2010, this issue). doi:10.1007/s00199-009-0437-1

Turocy, T. L.: Computing sequential equilibria using agent quantal response equilibria. Econ Theory $\mathbf{4 2}$ (2010, this issue). doi:10.1007/s00199-009-0443-3

van den Elzen, A.H., Talman, A.J.J.: A procedure for finding Nash equilibria in bi-matrix games. ZOR Methods Models Oper Res 35, 27-43 (1991)

von Stengel, B.: Efficient computation of behavior strategies. Games Econ Behav 14, 220-246 (1996)

Wilson, R.: Computing equilibria of $N$-person games. SIAM J Appl Math 21, 80-87 (1971)

Wilson, R.: Computing equilibria of two-person games from the extensive form. Manage Sci 18, $448-460(1972)$ 